\title{
Identification of loci affecting teat number by genome-wide association studies on three pig populations
}

\author{
Jianhong Tang', Zhiyan Zhang', Bin Yang', Yuanmei Guo', Huashui Ai ', Yi Long', Ying Su', Leilei Cui', Liyu Zhou', \\ Xiaopeng Wang ${ }^{1}$, Hui Zhang ${ }^{1}$, Chengbin Wang ${ }^{1}$, Jun Ren ${ }^{1}$, Lusheng Huang', and Nengshui Ding ${ }^{1}$ **
}

* Corresponding Author: Nengshui Ding
Tel: +86-791-83813080, Fax: +86-791-83805967,
E-mail: dingyd2005@hotmail.com

'State Key Laboratory for Pig Genetic Improvement and Production Technology, Jiangxi Agricultural University, Nanchang 330045, China

Submitted Nov 30, 2015; Revised Feb 6, 2016; Accepted Mar 25, 2016
Objective: Three genome-wide association studies (GWAS) and a meta-analysis of GWAS were conducted to explore the genetic mechanisms underlying variation in pig teat number. Methods: We performed three GWAS and a meta-analysis for teat number on three pig populations, including a White Duroc $\times$ Erhualian $\mathrm{F}_{2}$ resource population $(\mathrm{n}=1,743)$, a Chinese Erhualian pig population $(\mathrm{n}=320)$ and a Chinese Sutai pig population $(\mathrm{n}=383)$. Results: We detected 24 single nucleotide polymorphisms (SNPs) that surpassed the genomewide significant level on Sus Scrofa chromosomes (SSC) 1, 7, and 12 in the $\mathrm{F}_{2}$ resource population, corresponding to four loci for pig teat number. We highlighted vertnin (VRTN) and lysine demethylase $6 \mathrm{~B}(K D M 6 B)$ as two interesting candidate genes at the loci on SSC7 and SSC12. No significant associated SNPs were identified in the meta-analysis of GWAS.

Conclusion: The results verified the complex genetic architecture of pig teat number. The causative variants for teat number may be different in the three populations

Keywords: Genome-wide Association Study, Imputation, Meta-analysis, Pig, Teat Number

\section{INTRODUCTION}

In pigs, nipples are essential for nursing babies during lactation. For a given number of piglets, the sows with more functional teats produce more weaned pigs as compared with those having fewer functional teats. Thereby, teat number is an economically important trait that significantly affects reproduction efficiency in pigs.

Unlike most reproductive traits, teat number is an inborn trait that is less affected by environmental factors [1]. Different pig breeds have variable teat, numbers, ranging from 8 to 25 . The genetic architecture of teat number is very complicated. To date, 120 quantitative trait loci (QTLs) have been identified for teat number which span almost all pig chromosomes (http://www.animalgenome.org/cgi-bin/QTLdb/SS/index). Recently, genome-wide association studies (GWAS) have been conducted to map loci affecting pig teat number and have identified 11 interesting candidate genes for this trait [2-4]. However, the responsible genes and causative mutations for the identified loci remain to be characterized. Moreover, QTLs for pig teat number in a broad context of diverse breeds warrant further investigations by using the advanced GWAS tool.

In this study, we conducted GWAS to map loci affecting teat number on three pig populations, including a White Duroc $\times$ Erhualian $\mathrm{F}_{2}$ resource population $(\mathrm{n}=1,743)$, a Chinese Erhualian population $(n=320)$ and a Chinese Sutai population $(n=383)$. The results improve our understanding of the genetic architecture of pig teat number, and provide novel molecular 
markers for the genetic improvement of this reproductive trait in the pig industry.

\section{MATERIALS AND METHODS}

\section{Animals and phenotypic recording}

This study was conducted strictly in compliance with guidelines of experimental animals established by the Ministry of Agriculture of China, and was approved by the ethics committee of Jiangxi Agricultural University (China).

Experiment animals were from three pig populations, including the White Duroc $\times$ Erhualian $\mathrm{F}_{2}$ resource population, Chinese Erhualian and Sutai populations. The $\mathrm{F}_{2}$ resource population was established by intercrossing 2 White Duroc boars (PIC 1075) and 17 Erhualian sows as described previously [5]. In brief, a total of $1,912 \mathrm{~F}_{2}$ pigs were generated by $9 \mathrm{~F}_{1}$ boars and $59 F_{1}$ sows in 6 batches, avoiding full-sub mating. We recorded $1,743 \mathrm{~F}_{2}$ pigs for teat number. Erhualian is a highly prolific Chinese indigenous pig breed characterized by its favorable maternity and high litter size. We brought 320 unrelated (no common ancestors within 3 generations) Erhualian pigs from an Erhualian conservation farm in Jiangsu province. Sutai is a Chinese cultivated pig breed that was originally derived from a cross between Chinese Taihu and Duroc pigs and have experienced artificial selection for pork production and fertility for more than 18 generations. A total of 383 Sutai pigs from 5 sires and 60 dams were used in this study. All animals from the three populations were raised indoor in the experimental farm of Jiangxi Agricultural University (China), and were recorded for teat number by simply counting right and left teat number at the weaning period.

\section{Genotyping and quality control}

A standard phenol/chloroform approach was used to extract DNA from ear tissues. DNA quantity and quality were determined by a Nanodrop-1000 spectrophotometry (Thermo Fisher, Waltham, MA, USA). All DNA samples were diluted to a final concentration of $50 \mathrm{ng} / \mathrm{mL}$. In our previous study, $1,899 \mathrm{~F}_{2}$ pigs were genotyped for 183 microsatellite markers [6]. Here, 928 $\mathrm{F}_{2}$ pigs and their $\mathrm{F}_{1}$ and $\mathrm{F}_{0}$ ancestors, 320 Erhualian and 383 Sutai pigs were genotyped for 62193 single nucleotide polymorphisms (SNPs) on the Illumina Porcine 60K Beadchip [7]. The $60 \mathrm{~K}$ SNP data of the $928 \mathrm{~F}_{2}$ pigs and their $\mathrm{F}_{0}$ and $\mathrm{F}_{1}$ ancestors (reference animals) were further employed to impute the $60 \mathrm{~K}$ SNP genotypes of $815 \mathrm{~F}_{2}$ pigs (target animals) that were not genotyped by $60 \mathrm{~K}$ SNP chip by using the following approach [8]. Firstly, haplotypes of the reference and target animals were partially reconstructed based on linkage and Mendelian segregation rules with the 183 microsatellite data using DualPHASE [9]. Secondly, haplotypes of the reference individuals were fully reconstructed with microsatellite data and 60K SNPs data using DAGPHASE, which was iteratively called by Beagle [9]. Last- ly, missing 60K SNP genotypes of the target pigs were filled via CHROMIBD, in which the linkage and a Markov model were utilized to estimate identity-by-descent (IBD) probabilities between target and parent chromosomes from the genotyped ancestors [10].

The quality control for real and imputed genotypes SNPs were carried out by the Plink 1.07 software [11]. Animals with SNP call rates $\leq 0.9$, SNPs with call rates $\leq 0.9$, minor allele frequencies (MAF) $\leq 0.05$, p-values $\leq 10-6$ for the Hardy-Weinberg disequilibrium test and Mendelian error rate $\geq 0.1$ were removed for further statistical analysis. A final set of 31,557, 27,719 and 43,998 SNPs were remained for GWAS in the $F_{2}$, Erhualian and Sutai populations, respectively.

\section{Statistical analysis}

A principal component analysis (PCA) was conducted to test population structures of the three populations. We generated the PCA plots by using the -mds-plot and -cluster options in Plink and visualized it via the R program. The GWAS was performed with a linear mixed model in GenABEL v1.8, a library of $\mathrm{R}$ program [12]. The GWAS model is shown as below:

$$
y=X \beta+S \alpha+Z u+e
$$

Where y represents the vector of phenotypes of all tested pigs; $\beta$ is the fixed effects, including all grand mean and sex; $\alpha$ is the regression coefficient of substituting allele; $\mathrm{u}$ is the vector of polygenic effect; $\mathrm{e}$ is the vector of residual errors. $\mathrm{X}$ and $\mathrm{Z}$ are incidence matrices for $\beta$ and $\mathrm{u}$, respectively. $\mathrm{S}$ is the number of substituting alleles.

$\mathrm{Q}$-values were computed by the q-value package of $\mathrm{R}$ language and were then used to estimate the false discovery rate (FDR) threshold for the significant p-value of $0.05[13,14]$. Confidence regions were determined by log of odds ratio (LOD) dropoff 2 from the peak SNPs. A meta-analysis of GWAS was performed to combine evidences of true associations from the three populations with appropriate weight via METAL [15]. The meta-analysis was implemented with an inverse variance based strategy that weighted on the $\beta$-coefficients [15]. A common set of 16,396 SNPs across these populations were used in the meta-analysis.

\section{Annotation of candidate genes}

The porcine genome assembly 10.2 (http://www.animalgenome.org/repository/pig/Genome_build_10.2_mapping/) and National Center for Biotechnology Information (NCBI) Genome (http://www.ncbi.nlm.nih.gov/genome/?term=pig) were retrieved to characterize candidate genes in targeted regions. Function and signal pathway of annotated candidate genes were found via Ensembl Biomart (http://www.biomart. org/), GeneCards (http://www.genecards.org/), NCBI Gene (http://www.ncbi.nlm.nih.gov/gene/) and Cellsignal (http:// 
www.cellsignal.com/).

\section{RESULTS}

\section{Phenotypic data and population stratification}

Descriptive statistics of teat number in the three tested populations are shown in Table 1. The total teat number of Erhualian pigs ranged from 14 to 25 , with an average number of 20.38 . In contrast, Sutai pigs had the lowest average number of teats (15.20). The PCA analysis indicated that no population stratification existed between the $928 \mathrm{~F}_{2}$ pigs with real $60 \mathrm{~K}$ SNP genotypes and the $815 \mathrm{~F}_{2}$ pigs with the imputed data in the White Duroc $\times$ Erhualian $F_{2}$ population, suggesting the reliability of the imputation method. Nevertheless, the $\mathrm{F}_{2}$, Erhualian and Sutai populations displayed apparently different population structures, reflecting their divergent genetic background (Supplementary Figure 1).

\section{GWAS results}

The average inflation factors $(\lambda)$ of GWAS ranged from 0.961 to 1.028 , indicating that all population structures were properly adjusted (Supplementary Figure 2). In total, we detected 24 SNPs associated with porcine teat number in the $\mathrm{F}_{2}$ resource population, including the loci around $295.04 \mathrm{Mb}$ (293.78 to $301.56 \mathrm{Mb}$ ) on Sus Scrofa chromosomes (SSC) 1, around $103.91 \mathrm{Mb}(89.15$ to $106.91 \mathrm{Mb})$ on SSC7, around $26.02 \mathrm{Mb}$
Table 1. Descriptive statistics of total teat number in three pig populations

\begin{tabular}{lcccccc}
\hline Populations & No. & Mean & SD & Max & Min & CV(\%) \\
\hline $\mathrm{F}_{2}{ }^{1)}$ & 1,743 & 17.17 & 1.42 & 22 & 13 & 8.27 \\
Erhualian & 320 & 20.38 & 1.66 & 25 & 14 & 8.16 \\
Sutai & 383 & 15.20 & 1.09 & 18 & 12 & 7.18 \\
\hline
\end{tabular}

SD, standard deviation; CV, coefficient of variance.

${ }^{1)}$ The White Duroc $\times$ Erhualian F2 resource population.

(26.02 to $31.88 \mathrm{Mb}$ ) and $55.88 \mathrm{Mb}(45.75$ to $56.79 \mathrm{Mb})$ on SSC12 (Table 2 and Figure 1). No significant association was found in the Erhualian and Sutai populations (Figure 1). Besides the above-mentioned significant loci, we noted that the SNPs around $25.30 \mathrm{Mb}$ on SSC1 and $42.95 \mathrm{Mb}$ on SSC6 in Erhualian pigs, and those around $3.51 \mathrm{Mb}$ on SSC7 and 135.71 $\mathrm{Mb}$ on SSC15 in Sutai pigs showed potential associations with p-values close to the significant threshold (Figure 1). The loci on SSC1 and SSC6 in Erhualian pigs has also been reported to be associated with teat number in a Meishan $\times$ Gottingen cross population and a Large White $\times$ Meishan $\mathrm{F}_{2}$ population [16, 17]. In the meta-analysis of GWAS, no SNP showed significant association with teat number (Table 2 and Figure 1). Ten SNPs reached the 0.20 q-value threshold, suggesting a minimum of $\sim 19 \%$ FDR for these SNPs if judged as significant ones.

\section{DISCUSSION}

Table 2. Significant loci for teat number identified in the White Duroc $x$ Erhualian $\mathrm{F}_{2}$ resource population

\begin{tabular}{|c|c|c|c|c|c|c|c|}
\hline $\mathrm{Chr}$ & Significant SNP & Position (bp) & $p$-value & Allele & MAF & Effect $\pm S E$ & Candidate gene \\
\hline 1 & CASI0009856 & 295043824 & $1.98 \times 10^{-5}$ & $A / G$ & 0.49 & $0.399 \pm 0.094$ & \\
\hline 1 & INRA0007578 & 297186811 & $3.38 \times 10^{-5}$ & $\mathrm{G} / \mathrm{A}$ & 0.49 & $0.393 \pm 0.095$ & \\
\hline 1 & DRGA0002521 & 297585240 & $2.71 \times 10^{-5}$ & $\mathrm{G} / \mathrm{A}$ & 0.49 & $0.397 \pm 0.095$ & \\
\hline 1 & ALGA0010388 & 298117818 & $2.78 \times 10^{-5}$ & $G / A$ & 0.49 & $0.394 \pm 0.094$ & \\
\hline 1 & MARC0096868 & 298139740 & $2.64 \times 10^{-5}$ & $\mathrm{~A} / \mathrm{G}$ & 0.49 & $0.395 \pm 0.094$ & \\
\hline 1 & ALGA0010391 & 298158057 & $2.78 \times 10^{-5}$ & $\mathrm{~A} / \mathrm{G}$ & 0.49 & $0.394 \pm 0.094$ & \\
\hline 1 & H3GA0004878 & 298951152 & $3.74 \times 10^{-5}$ & $\mathrm{C} / \mathrm{A}$ & 0.49 & $0.382 \pm 0.092$ & \\
\hline 1 & H3GA0004881 & 298972575 & $3.74 \times 10^{-5}$ & $A / G$ & 0.49 & $0.382 \pm 0.092$ & \\
\hline 1 & ALGA0010455 & 300316974 & $3.82 \times 10^{-5}$ & $\mathrm{C} / \mathrm{A}$ & 0.49 & $0.372 \pm 0.091$ & \\
\hline 7 & H3GA0022664 & 103910821 & $3.23 \times 10^{-5}$ & $\mathrm{G} / \mathrm{A}$ & 0.39 & $0.336 \pm 0.083$ & VRTN \\
\hline 12 & MARC0049315 & 26022727 & $6.07 \times 10^{-6}$ & $\mathrm{G} / \mathrm{A}$ & 0.43 & $0.314 \pm 0.072$ & \\
\hline 12 & MARC0074514 & 26539746 & $2.06 \times 10^{-5}$ & $\mathrm{~A} / \mathrm{G}$ & 0.38 & $0.309 \pm 0.076$ & \\
\hline 12 & DIAS0001462 & 26604209 & $1.95 \times 10^{-5}$ & $A / G$ & 0.38 & $0.310 \pm 0.076$ & \\
\hline 12 & ALGA0065766 & 27027068 & $1.45 \times 10^{-5}$ & $\mathrm{G} / \mathrm{A}$ & 0.38 & $0.315 \pm 0.076$ & \\
\hline 12 & DIAS0004287 & 27169632 & $1.38 \times 10^{-5}$ & $\mathrm{~A} / \mathrm{G}$ & 0.38 & $0.316 \pm 0.076$ & \\
\hline 12 & MARC0022385 & 27272823 & $1.38 \times 10^{-5}$ & $\mathrm{G} / \mathrm{A}$ & 0.38 & $0.316 \pm 0.076$ & \\
\hline 12 & ALGA0105911 & 27302587 & $1.38 \times 10^{-5}$ & $\mathrm{~A} / \mathrm{C}$ & 0.38 & $0.316 \pm 0.076$ & \\
\hline 12 & ALGA0065836 & 28302694 & $3.08 \times 10^{-5}$ & $C / A$ & 0.38 & $0.304 \pm 0.076$ & \\
\hline 12 & ALGA0065906 & 31610473 & $2.37 \times 10^{-5}$ & $\mathrm{G} / \mathrm{A}$ & 0.38 & $0.308 \pm 0.076$ & \\
\hline 12 & ASGA0102838 & 55575876 & $2.44 \times 10^{-5}$ & $\mathrm{G} / \mathrm{A}$ & 0.24 & $0.313 \pm 0.075$ & \\
\hline 12 & ALGA0121951 & 55883014 & $4.89 \times 10^{-6}$ & $\mathrm{G} / \mathrm{A}$ & 0.35 & $0.326 \pm 0.071$ & KDM6B \\
\hline 12 & ALGA0066981 & 56035133 & $2.90 \times 10^{-5}$ & $\mathrm{C} / \mathrm{A}$ & 0.50 & $0.376 \pm 0.089$ & \\
\hline 12 & ALGA0066986 & 56093886 & $3.26 \times 10^{-5}$ & $\mathrm{G} / \mathrm{A}$ & 0.50 & $0.376 \pm 0.089$ & \\
\hline 12 & H3GA0034753 & 56111732 & $3.63 \times 10^{-5}$ & $\mathrm{~A} / \mathrm{G}$ & 0.50 & $0.374 \pm 0.089$ & \\
\hline
\end{tabular}

MAF, minor allele frequencies; $S E$, standard error; VRTN, vertnin; KDM6B, lysine demethylase 6B. 

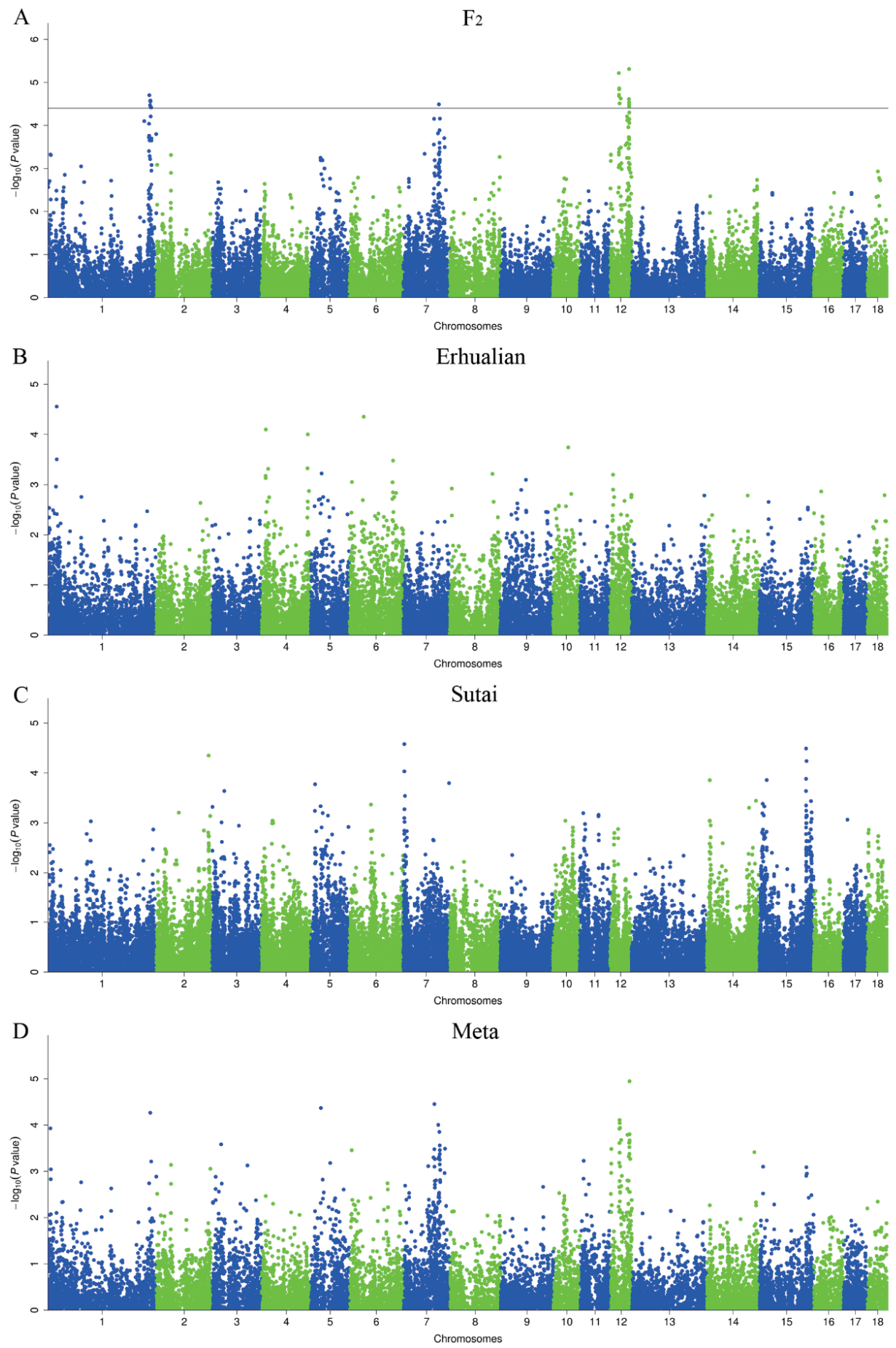

Figure 1. Manhattan plots of genome-wide association studies for pig teat number. GWAS were conducted on the White Duroc $\times$ Erhualian $\mathrm{F}_{2}$ resource population (A), Erhualian pigs (B), Sutai pigs (C) and the meta-analysis (D). Negative log10 p-value of all SNPs are plotted against their genomic positions. SNPs on different chromosomes are denoted with different colors. The dashed lines represent the 0.05 q-value genome-wide thresholds. GWAS, genome-wide association studies; SNPs, single nucleotide polymorphisms.

\section{Genotype imputation and meta-analysis for GWAS}

More currently, genotype imputation and meta-analysis have been explored in GWAS of genetic diseases or phenotypic traits $[15,18,19]$. Genotype imputation is a reliable tool to equate different sets of markers from multiple platforms [18]. With the development and improvement of multiple imputation al- gorithms, missing genotypes can be imputed with high accuracy rates [20]. In the White Duroc $\times$ Erhualian $\mathrm{F}_{2}$ resource population, we have estimated an average of $91.44 \%$ of genotype concordance, $95.36 \%$ of allelic concordance and 0.85 of $\mathrm{r}^{2}$ correlation between real and imputed data using a cross-validation analysis [21]. By applying an imputation based GWAS, we 
have successfully identified a list of significant loci for traits related to male reproduction in this $\mathrm{F}_{2}$ population [21]. Meta-analysis of GWAS provides a robust tool to identify true assoc-iations by pooling data from different studies or populations [15]. This approach enables researchers to efficiently account for population substructure, studies-specific covariates, and individual relatedness [15].

In this study, we implemented the imputation method to deduce the $60 \mathrm{~K}$ SNP genotypes of $815 \mathrm{~F}_{2}$ pigs using the genotyped $928 \mathrm{~F}_{2}$ pigs and their $\mathrm{F}_{0} / \mathrm{F}_{1}$ ancestor as the reference panel. The markers on sex chromosomes were not imputed for a lack of an appropriate algorithm [22]. This imputation approach allowed us to increase the sample size up to 1,743 animals in the $\mathrm{F}_{2}$ resource population. In the meta-analysis of GWAS, we did not identify any SNP that surpassed the significant threshold. One explanation could be that different popul-ations have distinct causative mutations for teat number; thus, the metaanalysis cannot increase but decreases the detection power. Another possible reason is that the qualified SNPs in the meta-analysis may be in different linkage disequilibrium phases with causative mutations in different populations, leading to inconsistent allelic directions and consequently reducing the detection power.

\section{Comparison of GWAS loci with previously identified QTLS}

Teat number is a complex reproductive trait. A total of 120 QTLs have been identified for this trait on almost all pig chromosomes except for SSC13, suggesting that teat number is controlled by multiple genes with generally small effects. We have previously identified 11 genome-wide significant QTLs for teat number by a genome scan on the White Duroc $\times$ Erhualian $\mathrm{F}_{2}$ resource population [6]. These loci were distributed on SSC1, 3, 4, 5, 6, 7, 8, and 12, which were consistent with previous reports [1,23-29]. Of the 11 significant loci, the loci on SSC1, SSC7 and SSC12 were replicated in this study (Table 2 and Figure 1). We note that the loci around 102 to $105 \mathrm{Mb}$ on SSC7 and around 52 to $56 \mathrm{Mb}$ on SSC12 were also consistently detected in a Landrace-based line and a Large White line $[2,3]$. However, those QTLs on SSC3, SSC4, SSC5, SSC6, and SSC8 that we detected in our previous study were not evidenced by the GWAS in this study. This may be due to the following points. First, the GWAS thresholds including Bonferroni-corrected and FDR-defined thresholds are more stringent than the QTL threshold. Second, the traditional QTL mapping conducts stepwise conditional analysis, i.e. the effect of QTL detected in a previous round was treated as a fixed effect in the statistical model when searching for QTL in the next round. This approach has smaller error residuals and consequently has a stronger detection power than the linear mixed GWAS model [30]. Third, only additive effect were included in our GWAS model while both additive and dominant effects were consid- ered in the QTL model. For example, the QTL on SSC6 has been identified as a significant locus for teat number with both additive and dominant effects in the $\mathrm{F}_{2}$ population [6]. Finally, the underlying assumptions of QTL and GWAS were not same. In the QTL mapping study, the QTL was assumed to be fixed in alternative alleles in the two found breeds, respectively, and the markers of three generations (founders, $F_{1}$ and $\mathrm{F}_{2}$ ) were used to track the QTL genotype in the $\mathrm{F}_{2}$ animals; while the SNP genotypes were considered as causative SNP genotypes in GWAS. Although we detected few significant loci in the GWAS, we obtained important clues for the most likely locations of these loci by using high-density SNPs across the genome as compared to sparse microsatellite makers used in the QTL mapping (see below). We note that two regions are associated with teat number on SSC12 where only one QTL was identified in our previous study [6]. This could be due to the fact that only 7 microsatellite markers with an average interval distance of $9.44 \mathrm{Mb}$ on this chromosome were used for mapping QTLs, which profoundly limited the mapping resolution of the traditional QTL analysis.

\section{Comparison of significant loci across the three tested populations}

We did not identify any significant locus that was shared by the three tested populations or two of these populations (Figure 1). Although the $F_{2}$ population was originally derived from a cross between White Duroc and Erhualian breeds, the four significant loci detected in the $\mathrm{F}_{2}$ population did not appear in the purebred Erhualian population. These loci are likely caused by variants that are alternatively fixed (or nearly fixed) in Duroc and Erhualian pigs. Sutai pigs are descendants of a hybrid between Duroc and Taihua (Erhualian is a subgroup of Taihu pigs), mimicking the genetic makeup of the $\mathrm{F}_{2}$ population. No common significant locus was evidenced between the two populations, either. These observations further reflected the complex genetic architecture of pig teat number. It should be mentioned that MAF of many chip SNPs varied considerably in the three populations. For example, all nine significant SNPs on chromosome 1 were fixed in the Erhualian population (data not shown). Hence, we cannot rule out the possibility of false positive or negative results that are caused by variable MAF in this study.

\section{Plausible candidate genes at the significant loci}

For significant loci that we identified, we searched plausible candidate genes within the confidence region of each locus. Combining our results with the earlier publications, we propose lysine demethylase $6 \mathrm{~B}(K D M 6 \mathrm{~B})$ and vertnin (VRTN) as two interesting candidate genes for the loci on SSC12 and SSC7. No annotated gene was found in the other QTL regions that are functionally related to the development of teat. $K D M 6 B$ is $0.42 \mathrm{Mb}$ away from the top SNP (ALGA0121951) at the genome- 
wide significant locus on SSC12. This gene plays a critical role in guiding the expression of T-box gene family in embryonic stem cell differentiation [31]. T-box3, a member of the T-box gene family, has been reported to influence the development of mammary gland placodes via interacting with Wnt family member 10 (Wnt10), lymphoid enhancer binding factor 1 (lef-1), and fibroblast growth factor 10 ( Fgf10) [32-34]. Moreover, Tbox 3 variants have been implicated in the Ulnar-Mammary Syndrome in humans, a genetic disease characterized by nipple and breast hypoplasia or aplasia $[35,36]$. Given that $K D M 6 B$ is located in the confidence interval of the genome-wide significant locus and has a potential role in the development of nipples, we highlight it as an interesting candidate gene for pig teat number that warrant further investigations.

VRTN is $0.71 \mathrm{Mb}$ away from the top SNP (H3GA0022664) at the genome-wide significant locus on SSC7 in the White Duroc $\times$ Erhualian $\mathrm{F}_{2}$ resource population (Figure 1). This gene is known to play an essential role in cell differentiation of embryos in different species and has a major effect on the development of thoracic vertebrae (ribs) in pigs $[37,38]$. Vertebral number is positively correlated with body size in pigs. Hogs with more vertebrae tend to have longer body length and better meat production capacity. Selective breeding for enlarging body size to increase meat production has been carried out for decades in Western commercial breeds, resulting in increased vertebral number and presumably more teats [3]. Therefore, VRTN has been selected as a promising candidate gene for the number of nipples by several other research groups $[2,3]$. Here, we observed that teat number is significantly correlated with vertebral number in the $\mathrm{F}_{2}$ population $(r=0.21$, $\mathrm{p}$-value $=1.51 \times 10-10)$. When conditional on the effect of a candidate causative mutation of VRTN (g.20311_20312ins29) $[37,38]$ in the $\mathrm{F}_{2}$ population, the GWAS signal at the SSC7 locus vanished in this population (data not shown). Hence, we assume that causative mutation(s) in the VRTN gene may have pleiotropic effects on the teat number and vertebral number. Recently, we shown that the VRTN mutation is significantly associated with teat number in a large sample of Western $\mathrm{Du}$ roc and Landrace pigs [39]. This finding has an immediate transition into the pig breeding schemes, providing a novel tool to improve both teat number and vertebral number by selection of the desirable allele at the VRTN locus. We note that no significant association signal was detected in the VRTN region in the Erhualian and Sutai populations. One reasonable explanation is that the causative variant(s) in the VRTN gene is fixed or very rare in the two populations.

\section{CONFLICT OF INTEREST}

We certify that there is no conflict of interest with any financial organization regarding the material discussed in the manuscript.

\section{ACKNOWLEDGMENTS}

This research was supported by National Basic Research Program (2014CB138501), Program for New Century Excellent Talents in University, the earmarked fund for Jiangxi Agriculture Research System (JXARS-03) and The National Key Technology R \& D Program (2011BAD28B01).

\section{REFERENCES}

1.Bidanel JP, Rosendo A, Iannuccelli N, et al. Detection of quantitative trait loci for teat number and female reproductive traits in Meishan x Large White $\mathrm{F}_{2}$ pigs. Animal 2008;2:813-20.

2.Lopes MS, Bastiaansen JW, Harlizius B, Knol EF, Bovenhuis H. A genome-wide association study reveals dominance effects on number of teats in pigs. PLoS One 2014;9:e105867.

3.Duijvesteijn N, Veltmaat JM, Knol EF, Harlizius B. High-resolution association mapping of number of teats in pigs reveals regions controlling vertebral development. BMC Genomics 2014;15:542.

4.Lee JB, Jung EJ, Park HB, et al. Genome-wide association analysis to identify SNP markers affecting teat numbers in an $\mathrm{F}_{2}$ intercross population between Landrace and Korean native pigs. Mol Biol Rep 2014;41:7167-73.

5.Guo T, Ren J, Yang K, Ma J, Zhang Z, Huang L. Quantitative trait loci for fatty acid composition in longissimus dorsi and abdominal fat: results from a White Duroc $\times$ Erhualian intercross $F_{2}$ population. Anim Genet 2009;40:185-91.

6.Ding N, Guo Y, Knorr C, et al. Genome-wide QTL mapping for three traits related to teat number in a White Duroc $\mathrm{x}$ Erhualian pig resource population. BMC Genet 2009;10:6.

7.Ramos AM, Crooijmans RP, Affara NA, et al. Design of a high density SNP genotyping assay in the pig using SNPs identified and characterized by next generation sequencing technology. PloS one 2009; 4:e6524.

8.Zhang Z, Druet T. Marker imputation with low-density marker panels in Dutch Holstein cattle. J Dairy Sci 2010;93:5487-94.

9.Druet T, Georges M. A hidden Markov model combining linkage and linkage disequilibrium information for haplotype reconstruction and quantitative trait locus fine mapping. Genetics 2010;184:78998.

10. Druet T, Farnir FP. Modeling of identity-by-descent processes along a chromosome between haplotypes and their genotyped ancestors. Genetics 2011;188:409-19.

11. Purcell S, Neale B, Todd-Brown K, et al. PLINK: a tool set for wholegenome association and population-based linkage analyses. Am J Hum Genet 2007;81:559-75.

12. Aulchenko YS, Ripke S, Isaacs A, Van Duijn CM. GenABEL: an R library for genome-wide association analysis. Bioinformatics 2007;23:1294-6.

13. Storey JD. A direct approach to false discovery rates. J R Stat Soc 2002;64:479-98.

14. Storey JD, Tibshirani R. Statistical significance for genomewide 
studies. Proc Natl Acad Sci USA 2003;100:9440-5.

15. Willer CJ, Li Y, Abecasis GR. METAL: fast and efficient metaanalysis of genomewide association scans. Bioinformatics 2010; 26:2190-1.

16. Hernandez S, Finlayson H, Ashworth C, Haley C, Archibald A. A genome-wide linkage analysis for reproductive traits in $\mathrm{F}_{2}$ Large White $\times$ Meishan cross gilts. Anim Genet 2014;45:191-7.

17. Wada Y, Akita T, Awata T, et al. Quantitative trait loci (QTL) analysis in a MeishanxGottingen cross population. Anim Genet 2000;31: 376-84.

18. Hao K, Chudin E, McElwee J, Schadt EE. Accuracy of genomewide imputation of untyped markers and impacts on statistical power for association studies. BMC Genet 2009;10:27.

19. Pei YF, Li J, Zhang L, Papasian CJ, Deng HW. Analyses and comparison of accuracy of different genotype imputation methods. PLoS One 2008;3:e3551.

20. Marchini J, Howie B. Genotype imputation for genome-wide association studies. Nat Rev Genet 2010;11:499-511.

21. Zhao X, Zhao K, Ren J, et al. An imputation-based genome-wide association study on traits related to male reproduction in a White Duroc x Erhualian F population. Anim Sci J 2015.

22. Wise AL, Gyi L, Manolio TA. eXclusion: toward integrating the $\mathrm{X}$ chromosome in genome-wide association analyses. Am J Hum Genet 2013;92:643-7.

23. Guo YM, Lee G, Archibald A, Haley C. Quantitative trait loci for production traits in pigs: a combined analysis of two Meishan $\times$ Large White populations. Anim Genet 2008;39:486-95.

24. Tortereau F, Gilbert H, Heuven HC, Bidanel JP, Groenen MA, Riquet J. Combining two Meishan $\mathrm{F}_{2}$ crosses improves the detection of QTL on pig chromosomes 2, 4 and 6. Genet Sel Evol 2010;42: 42.

25. Holl J, Cassady J, Pomp D, Johnson R. A genome scan for quantitative trait loci and imprinted regions affecting reproduction in pigs. J Anim Sci 2004;82:3421-9.

26. Lee S, Chen Y, Moran C, et al. Linkage and QTL mapping for Sus scrofa chromosome 5. J Anim Breed Geneti2003;120:38-44.

27. Cassady JP, Johnson RK, Pomp D, et al. Identification of quantitative trait loci affecting reproduction in pigs. J Anim Sci 2001;79:62333.

28. Sato S, Atsuji K, Saito N, et al. Identification of quantitative trait loci affecting corpora lutea and number of teats in a Meishanx Duroc $\mathrm{F}_{2}$ resource population. J Anim Sci 2006;84:2895-901.

29. Hirooka H, de Koning DJ, Harlizius B, et al. A whole-genome scan for quantitative trait loci affecting teat number in pigs. J Anim Sci 2001;79:2320-6.

30. Guo YM, Zhang ZY, Ma JW, Ai HS, Ren J, Huang LS. A genomewide association study of feed efficiency and feeding behaviors at two fattening stages in a White DurocxErhualian F population. J Anim Sci 2015;93:1481-9.

31. Ohtani K, Zhao C, Dobreva G, et al. Jmjd3 controls mesodermal and cardiovascular differentiation of embryonic stem cells. Circ Res 2013;113:856-62.

32. Davenport TG, Jerome-Majewska LA, Papaioannou VE. Mammary gland, limb and yolk sac defects in mice lacking Tbx3, the gene mutated in human ulnar mammary syndrome. Development 2003; 130:2263-73.

33. Jerome-Majewska LA, Jenkins GP, Ernstoff E, Zindy F, Sherr CJ, Papaioannou VE. Tbx3, the ulnar-mammary syndrome gene, and Tbx2 interact in mammary gland development through a p19Arf/p53-independent pathway. Dev Dyn 2005;234:922-33.

34. Eblaghie MC, Song SJ, Kim JY, Akita K, Tickle C, Jung HS. Interactions between FGF and Wnt signals and Tbx3 gene expression in mammary gland initiation in mouse embryos. J Anat 2004; 205:1-13.

35. Rapini RP, Bolognia J, Jorizzo J. Dermatology, 2-Volume Set. St. Louis: Mosby, 2007: ISBN 1-4160-2999-0.

36. Klopocki E, Neumann LM, Tönnies H, Ropers H-H, Mundlos S, Ullmann R. Ulnar-mammary syndrome with dysmorphic facies and mental retardation caused by a novel $1.28 \mathrm{Mb}$ deletion encompassing the TBX3 gene. Eur J Hum Genet 2006;14:1274-9.

37. Mikawa S, Sato S, Nii M, et al. Identification of a second gene associated with variation in vertebral number in domestic pigs. BMC Genet 2011;12:5.

38. Fan Y, Xing Y, Zhang Z, et al. A further look at porcine chromosome 7 reveals VRTN variants associated with vertebral number in Chinese and Western pigs. PloS one 2013;8:e62534.

39. Yang J, Huang L, Yang M, et al. Possible introgression of the VRTN mutation increasing vertebral number, carcass length and teat number from Chinese pigs into European pigs. Sci Rep 2016;6. 